\section{Oral bacterial biofilms - history in progress}

Ask microbiologists what the word 'biofilm' brings to mind, and many would speak about Pseudomonas aeruginosa, about microtitre plates or flowcells, about 'mushrooms' and 'voids', and about antibiotic resistance and device-related infections. They would perhaps speak of biofilm growth as development: distinct phases or behaviours such as attachment, spreading and dispersal. They would almost certainly mention quorum sensing or communication, and almost all would use the word 'community' or 'population'. Yet, despite their well-founded enthusiasm and the numerous impressive examples they would cite, the populations to which they typically refer are in fact quite homogeneous; most of what is clearly recognized as biofilm research has been conducted in vitro using single bacterial pure cultures. However, working in relative obscurity and beginning several decades prior to popularization of the word biofilm, oral microbiologists established the paradigm for our understanding of development in realworld biofilm communities: dental plaque. Irene Dige and colleagues continue this tradition in the current issue of Microbiology by describing and quantifying the spatiotemporal population dynamics of Actinomyces naeslundii in early supragingival biofilms (Dige et al., 2009).

Historically, examination of biofilm growth at the level of single cells began in earnest when the elecron microscope became a common biological tool. Microbiologists and 'cariologists', especially those in Scandinavian dental schools, used this tool to study the most easily accessible biofilms of the human body: those that form on tooth surfaces. The biofilms were retrieved at sequential time points on small pieces of glass or bovine enamel that had been carried in the oral cavity of a volunteer: typically an eager(?) student. To isolate, count, and identify organisms after removal, the biofilm was scraped off, homogenized, and plated on various media. With the perfect hindsight that the oral cavity is home to some 800 phylotypes, and compounded by the relatively rudimentary speciation techniques at the time, this approach was laborious, duplicative, and often yielded datasets that were difficult to compare from laboratory to laboratory. However, one arrived at the conclusion that a lot of different bugs were present, and one could say that the great majority of those were streptococci, with other bacteria such as actinomyces also present. Carrier pieces were simultaneously examined with the electron microscope. Early on $(\leqslant 4 \mathrm{~h}$ of carrier wear), the biofilm consisted of small aggregates of spherical cells (generally no more than three or four cells) with the occasional non-coccoid morphotype thrown in. As time progressed, the total cell count as well as the size of the cell aggregates increased rapidly, in parallel with species diversity determined by plating. However, the electron microscope provided little information on the spatial arrangement of different bacterial species within the biofilm; cocci are cocci and rods are rods. Were the aggregates clonal, at least early on? Could the later, much bigger aggregates be mixtures of streptococci with any number of other coccoid organisms such as veillonellae? What is the arrangement of species after the aggregates join to confluence? A comb with finer teeth was needed.

The advent of immunofluorescence microscopy and FISH answered some of these questions. Clonality was out and communities were in, at least for the cocci.
Dige et al. (2009) confirm an observation from the early days: that a Gram-positive bacterium, thought to be an actinomyces based on plating results, was regularly found at the substratum. The authors use FISH, confocal microscopy and stereological enumeration to expand upon that initial observation. They show that $A$. naeslundii grows in a quasi-clonal manner, that its numbers increase slowly relative to those of streptococci, and that it occupies pockets within the biofilm that can always be followed to the substratum. Thus, A. naeslundii is not only an early colonizer of the tooth surface, but also demonstrates a patchy, mosaic-like distribution in older biofilms. These observations, together with the lack of single $A$. naeslundii cells at the top of older biofilms, suggest that the organism gets into the biofilm early through recognition of and adherence to streptococci, but then putters along on its own little patch of real estate. Part of this behaviour may be due to its ability to use the streptococcal fermentation product lactic acid as an energy source. Veillonellae share this ability and are also found intimately associated with streptococci (Palmer et al., 2006). Interestingly, Actinomyces oris, until recently known as A. naeslundii but now reclassified as a unique species (Henssge et al., 2009), does not grow in clumps but prefers to mix itself throughout the streptococcal biomass, at least when grown in saliva in vitro (Palmer et al., 2001). Could these different behaviours between closely related organisms indicate physiological traits which are detectable only when saliva is the nutrient source? Or only when appropriate partner organisms are present?

Microbiology Comment provides a forum for discussion of scientific issues arising directly from papers published in the journal. The authors of papers under discussion will be offered an opportunity to respond.

Guidelines on how to submit a Microbiology Comment article can be found in the Instructions for Authors at http://mic.sgmjournals.org

It should be noted that the Editors of Microbiology do not necessarily agree with the views expressed in Microbiology Comment.

Charles J. Dorman, Editor-in-Chief 
Such questions of the physiological basis for spatial distribution in natural biofilms are being explored in other ecosystems (Boetius et al., 2000), and the paradigm system of dental plaque presents many such opportunities.

\section{Robert J. Palmer}

National Institutes of Health/NIDCR, Bldg 30, Room 310, Bethesda, 20892, USA

Correspondence: Robert Palmer (rjpalmer@dir.nidcr.nih.gov)

\section{REFERENCES}

Boetius, A., Ravenschlag, K., Schubert, C. J., Rickert, D., Widdel, F., Gieseke, A., Amann, R., Jørgensen, B. B., Witte, U. \& Pfannkuche, O. (2000). A marine microbial consortium apparently mediating anaerobic oxidation of methane. Nature 407, 623-626.

Dige, I., Raarup, M. K., Nyengaard, J. R., Kilian, M. \& Nyvad, B. (2009). Actinomyces naeslundii in initial dental biofilm formation. Microbiology 155, 2116-2126.

Henssge, U., Do, T., Radford, D. R., Gilbert, S. C., Clark, D. \& Beighton, D. (2009). Emended description of Actinomyces naeslundii and descriptions of Actinomyces oris sp. nov. and Actinomyces johnsonii sp. nov., previously identified as Actinomyces naeslundii genospecies 1, 2 and WVA 963. Int J Syst Evol Microbiol 59, 509-516.

Palmer, R. J., Jr, Kazmerzak, K., Hansen, M. C. \& Kolenbrander, P. E. (2001). Mutualism versus independence: strategies of mixed-species oral biofilms in vitro using saliva as the sole nutrient source. Infect Immun 69, 5794-5804.

Palmer, R. J., Jr, Diaz, P. I. \& Kolenbrander, P. E. (2006). Rapid succession within the Veillonella population of a developing human oral biofilm in situ. J Bacteriol 188, 4117-4124.

DOI 10.1099/mic.0.030809-0

\section{$\mathrm{H}-\mathrm{NS}$ and genomic bridge building: lessons from the human pathogen Salmonella Typhi}

The H-NS protein has emerged as one of the leading causes of transcriptional repression in Gram-negative bacteria. In a paper published in this issue, De la Cruz and colleagues shed new light on the role of DNA curvature in the repressive mechanism, using a porin gene promoter from the human pathogen Salmonella
Typhi as their experimental system (De la Cruz et al., 2009). Their data help to deepen our understanding of the importance of local DNA structure in facilitating the interaction of $\mathrm{H}-\mathrm{NS}$ with its target sites in DNA and in establishing an effective nucleoprotein complex for the repression of transcription.

DNA curvature was described as being an important feature of H-NS binding sites many years ago (Yamada et al., 1990) but the reason for its importance remained obscure until recently. This is in spite of the considerable effort that has been expended in analysing H-NS interaction with regions of curvature, using both naturally occurring and artificial sequences with intrinsic curvature (Jordi et al., 1997; Rimsky et al., 2001). A requirement for A + T-rich DNA has also been noted, which is interesting in the light of the facts that (1) A+T-richness is a common feature of bacterial promoters, (2) an appropriate spacing of $\mathrm{A}+\mathrm{T}$-rich patches in B-DNA can impose curvature and (3) curved DNA is often found close to promoters (Barbic et al., 2003; Jauregui et al., 2003; Lang et al., 2007). The coincidence of these features seems to produce an ideal platform upon which $\mathrm{H}$ NS can repress the very large number of promoters that it is known to regulate. Central to the repression mechanism is the creation of DNA-H-NS-DNA bridges that impede transcription initiation (Dame et al., 2005; Dorman \& Kane, 2009).

De la Cruz et al. (2009) have examined the role of DNA static curvature in the promoter region of the ompS1 porin gene in $S$. Typhi. Bends were predicted in silico and detected by an electrophoretic technique in which the position of the bend centre relative to the ends of a DNA fragment results in a temperaturedependent alteration in the migration of the DNA molecule through a polyacrylamide gel. Impressively, the authors have been able to remove the bend by making just two changes to the base composition of the DNA, at positions -135 and -151 upstream of the ompS1 transcription start site $(+1)$. The presence of these base substitutions derepresses transcription of the promoter by about sevenfold. H-NS is known to bind to the ompS1 regulatory sequences, as is the paralogous protein StpA (De la Cruz et al.,
2007). The previously determined binding sites extend from the region of intrinsic curvature to the transcription start site. In this new study additional binding sites for $\mathrm{H}-\mathrm{NS}$ are identified upstream of the curve, raising the possibility that $\mathrm{H}-\mathrm{NS}$ may build bridges between the DNA sequences located upstream and downstream of the curve, resulting in the creation of a nucleoprotein complex that represses the ompS1 promoter. This is an intuitively appealing scenario that is consistent with earlier repression models that involve DNA-H-NS-DNA bridges (Prosseda et al., 2004). Interestingly, the suppression of DNA curvature in the ompS1 upstream regulatory region does not result in full derepression of the promoter; this requires inactivation of the $h n s$ gene as well. If the hns gene is inactivated but the curve is left intact (or is restored following mutation) a lower level of derepression results. This hints at the presence in the cell of another curve-dependent repressor of ompS1 transcription. StpA is an attractive candidate, not least because it shares so many properties with $\mathrm{H}-\mathrm{NS}$ and is known to regulate ompS1 transcription (De la Cruz et al., 2007).

The observation that $\mathrm{H}-\mathrm{NS}$ continues to exert a negative effect on ompS1 transcription in the absence of the curve suggests that $\mathrm{H}-\mathrm{NS}$ can still interact with the regulatory region DNA in the absence of bridging across the curve; electrophoretic mobility shift data support this. Of course these data do not rule out the possibility that H-NS may engage in highly localized bridging within each of the 'arms' of the regulatory region as well as more conventional binding and nucleation.

The ompS1 promoter is also under the control of other regulators, and the DNAH-NS-DNA bridging model provides a useful framework in which to consider their contributions at a mechanistic level. Prominent among these are the OmpR and LeuO DNA-binding proteins (De la Cruz et al., 2007; Flores-Valdez et al., 2003; Oropeza et al., 1999). Both are pleiotropic regulators of transcription. OmpR belongs to the response regulator protein family, most of whose members are transcription regulators that are subject to control by phosphorylation by histidine protein kinase sensor proteins (Kenney, 2002). LeuO is a member of the LysR family of 